Luca De Lucia. Catedrático de Derecho Administrativo Italiano y Europeo en la Università degli Studi di Salerno. Dentro de sus publicaciones más recientes, véanse: (con E. Schmidt-Assmann y M.C. Romano), "Prospettive della tutela giurisdizionale amministrativa in Germania e in Italia", en Diritto processuale amministrativo, 2016, pp. 711-769; "La conferenza di servizi nel decreto legislativo 30 giugno 2016", en Rivista giuridica di urbanistica, 127, 2016, pp. 12-14; (con P. Chirulli), "Specialized Adjudication in EU Administrative Law - The Boards of Appeal of EU Agencies", en European Law Review, 2015, pp. 832-857; "Il diritto amministrativo tedesco e il rinnovamento della dogmatica giuridica", en Rivista trimestrale di diritto pubblico, 2015, pp. 1-8.

Contacto:1delucia@unisa.it 


\section{CONFLICTO, DERECHO Y ECONOMÍA EN LA UNIÓN EUROPEA DESPUÉS DE LA CRISIS}

Luca De Lucia

Università degli Studi di Salerno

\section{CONFLICT, LAW AND ECONOMY IN THE EUROPEAN UNION AFTER THE CRISIS}

DOI: $1017450 / 160205$

Fecha de recepción 2 de agosto de 2016; fecha de aceptación 7 de septiembre de 2016. El artículo es fruto de un proyecto de investigación desarrollado en el Dipartimento di Scienze Politiche, Sociali e delle Comunicazioni, Università degli studi di Salerno.

\section{Resumen}

La crisis económica y financiera ha dado lugar a profundas transformaciones, relativas también a las relaciones entre la Unión Europea y los Estados miembros en el ámbito de la llamada gobernanza económica. Gracias a dos conceptos elaborados por Michel Foucault -el pastorado y el poder disciplinario-, este ensayo examina la naturaleza, el alcance y las consecuencias de algunos de esos cambios constitucionales. 


\section{Palabras clave}

Crisis, gobernanza económica, pastorado, poder disciplinario, cambios constitucionales.

\section{Abstract}

The economic and financial crisis has led to profound changes, which also concern relations between the European Union and its Member States in the area of so-called economic governance. Thanks to two concepts developed by Michel Foucault -the pastorate and the disciplinary power- this essay examines the nature, scope and consequences of some of these constitutional changes.

\section{Keywords}

Crisis, economic governance, pastorate, disciplinary power, constitutional changes. 


\section{Antiguas y nuevas técnicas de gobierno de la Unión Europea}

Las respuestas institucionales de la Unión Europea (UE) a la crisis han dado lugar a una nueva gobernanza económica en la que, por un lado, la injerencia de las instituciones europeas en las políticas económicas y de balance de los Estados miembros es mucho más evidente que en el pasado; por otro lado, la reglamentación jurídica adquiere un papel residual.

Para comprender el sentido de lo acaecido, cabe partir de una simple afirmación: el derecho (jurisprudencia, normas y principios generales) es un elemento esencial en la construcción europea. De hecho, la UE (y, antes que esta, la Comunidad Económica) es una "comunidad de derecho", en la que el juez europeo (y los nacionales) tiene un papel fundamental ${ }^{2}$. Por consiguiente, en la mayoría de los casos, los instrumentos de gobierno de la UE son actos jurídicos vinculantes (caracterizados también por la primacía y, a veces, por el efecto directo en relación con los ordenamientos nacionales). Además, el derecho desempeña un papel importante en la gestión del pluralismo institucional, de manera que los conflictos entre los sujetos se solucionen por medio de procedimientos legales (a menudo basados en una lógica deliberativa) ${ }^{3}$. De ahí que el carácter jurídico haya sido fundamental en el proceso de integración, tal y como se destaca en el famoso eslogan "integración a través del derecho", para el cual el derecho representa al mismo tiempo el objeto y el instrumento de la integración ${ }^{4}$.

Desde hace dos décadas, a este sistema se ha añadido progresivamente la llamada "nueva gobernanza", una expresión que se refiere al gobierno de determinados sectores (de competencia estatal) que emplean, sobre todo, instrumentos de soft law (aunque establecidos con medidas de hard law $)^{6}$. Son típicos de esta técnica la definición en el

1. Tribunal de Justicia, C-294/83, $\$ 23$.

2. Véase, F. C. Mayer, Europa als Rechtsgemeinschaft, en G. F. Schuppert, Ingolf Pernice, Ulrich Haltern (Hrsg.), Europawissenschaft Nomos, Paper 8, Walter Hallstein-Institut Paper, Baden-Baden, 2005.

3. C. Joerges, J. Neyer, "From Intergovernmental Bargaining to Deliberative Political Processes: The Constitutionalisation of Comitology”, en European Law Journal, Sweet \& Maxwell, Issue 3, 1997, pp. 273 ss.; C. Joerges, The Idea of a Three-Dimensional Conflicts Law as Constitutional Form, en Id., (ed.) Constitutionalism, Multilevel Trade Governance and International Economic Law, E.-U. Petersmann, Oxford, 2011, p. 413 ss.

4. J. H. H. Weiler, “The Community System: the Dual Character of Supranationalism”, en Yearbook of European Law, Oxford University Press, Oxford - New York, 1981, p. 267 ss.

5. K. Armstrong, "The Character of EU Law and Governance: From 'Community Method' to New Modes of Governance”, en Current Legal Problems, G. W. Keeton y G. Schwarzenberger (eds.), Faculty of Laws, University College, London, 2011, p. 179 ss.; M. Dawson, “Three Waves of New Governance in the European Union”, en European Law Review, Sweet \& Maxwell, United Kingdom, 2011, p. 208 ss.

6. D. M. Trubek, L. Trubek, "New Governance and Legal Regulation: Complementarity, Rivalry, and Transformation”, en Columbia Journal of European Law, Sweet \& Maxwell, United Kingdom, 2007, p. 539 ss.; K. Armstrong, The Character of EU Law, p. 179 ss. 
Consejo de objetivos que los Estados deben alcanzar, el empleo de instrumentos de medición de los resultados alcanzados (indicadores y benchmarking) y el intercambio de prácticas óptimas que la Comisión se ocupa de vigilar ${ }^{7}$.

En este contexto, adquiere un significado particular el método abierto de coordinación previsto en el Tratado de Maastricht para la gestión centralizada de la unión monetaria y para garantizar una duradera convergencia de los resultados económicos de los Estados $^{8}$. En su versión originaria, la gobernanza económica consistía en la "vigilancia multilateral" (basada en pareceres y recomendaciones de la Comisión y del Consejo) de las políticas económicas nacionales y en el control de los déficits públicos excesivos en que podía desembocar -una posibilidad que, por mucho tiempo, se ha considerado improbable ${ }^{9}$ - en sanciones a cargo del Estado insolvente.

\section{La respuesta europea a la crisis económica}

La UE y los Estados miembros adoptaron numerosas medidas tras la crisis de $2008^{10}$. No queremos detenernos en los detalles, pero es suficiente recordar la disciplina del semestre europeo $^{11}$, la que vigila los proyectos nacionales de balance (y aquella relativa al eventual procedimiento contra los déficits excesivos $)^{12}$ con los demás mecanismos introducidos por el llamado Fiscal Compact ${ }^{13}$, la de la prevención y corrección de los desequilibrios macroeconómicos ${ }^{14} \mathrm{y}$ del aumentado control en los Estados de la eurozona amenazados o afectados por crisis y dificultades financieras ${ }^{15}$. Con base en estas normativas, las instituciones europeas deben velar por las políticas nacionales económicas y de balance y por las condiciones económicas y financieras de los Estados.

7. J. Arrowsmith, K. Sisson y P. Marginson, “What Can 'Benchmarking' Offer the Open Method of Co-ordination?”, en Journal of European Public Policy, Routledge, London, 2004, p. 311 ss.

8. D. Hodson, Macroeconomic Co-ordination in the Euro Area: the Scope and Limits of the Open Method, en Journal of European Public Policy, 2004, p. 231 ss.; P. Craig, EU Administrative Law, Oxford University Press, Oxford, 2006, p. 195 ss.

9. D. Hodson y I. Maher, "Soft Law and Sanctions: Economic Policy Co-ordination and Reform of the Stability and Growth Pact”, en Journal of European Public Policy, Routledge, London, 2004, p. 798 ss.

10. Para una visión global, cfr. A. Hinarejos, The Euro Area Crisis in Constitutional Perspective, Oxford University Press, Oxford, 2015; K. Tuori, K. Tuori, The Eurozone Crisis: A Constitutional Analysis, Cambridge University Press, Cambridge, 2014.

11. Que incluye la evaluación de los programas nacionales de estabilidad y de convergencia acerca de la consecución de los objetivos de mediano plazo y de los programas nacionales de reforma: Reg. (CE) 1466/97 del Consejo del 7 de julio de 1997 (versión revisada).

12. Reg. 473/2013 del Parlamento europeo y del Consejo del 21 de mayo de 2013.

13. Tratado sobre la estabilidad, coordinación y gobernanza en la unión económica y monetaria suscrito por 25 Estados miembros de la Unión europea el 2 de marzo de 2012.

14. Reg. 1176/2011 del Parlamento europeo y del Consejo del 16 de noviembre de 2011.

15. Reg. 472/2013 del Parlamento europeo y del Consejo del 21 de mayo de 2013. 
El proceso decisional es gradual ${ }^{16}$ : un juicio negativo sobre un Estado puede implicar consecuencias proporcionales a las dificultades de la situación; cuanto peor es la evaluación, más laboriosas serán las intervenciones que el Estado deberá poner en práctica bajo el control de la Comisión y del Consejo. En los casos más graves, además de evaluar una serie de documentos (planes de acción colectivos, programas de cooperación económica), esas instituciones pueden ordenarle al Estado que adopte determinadas medidas (por ejemplo, el artículo 126, párr. 9, del TFUE), infligiéndole sanciones si no hace todo lo posible para solucionar el problema ${ }^{17}$. En realidad, todas estas normativas implican poderes de vigilancia de las instituciones (sobre todo de la Comisión) que sobrepasan los temas económicos, puesto que pueden afectar todas las políticas públicas nacionales.

A pesar de que, en esta materia, el contenido constitucional no ha cambiado desde el Tratado de Maastricht, el derecho derivado (sobre todo con los llamados six-pack y two-pack) y el llamado Fiscal Compact han tenido una influencia muy significativa sobre el precedente estado normativo. Por ejemplo, por mucho tiempo la disciplina sobre la coordinación de las políticas económicas (parcialmente diferente es el tema del procedimiento de corrección de los déficits excesivos) ha sido considerada fuente de compromisos solo políticos ${ }^{18}$. Hoy día la situación ha cambiado por una serie de poderes sancionatorios (incluso por hipótesis que el Tratado no previó) que el Consejo puede ejercer tras la propuesta de la Comisión, o de la regla de la llamada "mayoría inversa" que, en muchos casos, lleva a la casi automática aprobación de las propuestas de la Comisión por parte del Consejo ${ }^{19}$. De ahí que hoy la Comisión desarrolle una función fundamental. La posición de los Estados (sobre todo de los que tienen mayores dificultades) ha estado afectada por muchos cambios, debido a una actividad de vigilancia europea muy intensa aunque, en la mayoría de los casos, sigue fundamentándose en pareceres y recomendaciones, es decir, en actos no vinculantes, cuyo alcance jurídico es ambiguo.

\footnotetext{
16. Véase el artículo 6, Reg. 1466/97 y artículos 3 y ss.; Reg. 1467/97, del Consejo del 7 de julio de 1997 (versión revisada). Cfr. K. A. Armstrong, Differentiated Economic Governance and the Reshaping of Dominium-Law, en M. Adams, F. Fabbrini, P. Larouche (eds.), The Constitutionalization of European Budgetary Constraints, Hart Publishing, Oxford, 2014, pp. 63 ss. 17. Reg. 1173/2011 del Parlamento europeo y del Consejo del 16 de noviembre de 2011 y Reg. 1174/2011 del Parlamento europeo y del Consejo del 16 de noviembre de 2011.

18. Al respecto véase F. Amtenbrink, J. de Haan, "Economic governance in the European Union: Fiscal policy discipline versus flexibility", en Common Market Law Review, Kluwer Law International, Netherlands, 2003, p. 1075 ss.

19. Se trata de la regla según la cual algunas propuestas de la Comisión pueden ser rechazadas por el Consejo solo con determinadas mayorías: ver art. 4, párr. 2, 3 y 6, reg. 1173/11; art. 6, párr. 2, reg. 1466/97; art. 10, párr. 4 reg. 1176/11; art. 3, párr. 3, reg. 1174/11.
} 
Es más incisiva la injerencia en los Estados que gozan de asistencia financiera. Después de una primera fase caracterizada por cierta experimentación ${ }^{20}$, ahora la materia se basa en el reg. n. ${ }^{\circ} 472 / 13^{21}$. Con base en su artículo 7 , el Estado que pide ayuda financiera debe poner en práctica (junto con la Comisión, "que actúa junto con el BCE y, eventualmente, con el FMI") un programa de arreglos que el Consejo debe aprobar con mayoría cualificada. El programa tiene el objetivo de volver a establecer "una situación económica sana y sostenible y de restaurar plenamente la capacidad del Estado miembro interesado de autofinanciarse en los mercados financieros"; se desarrolla por medio de un Memorándum de entendimiento (MoU), firmado por el destinatario y por la Comisión, y que contiene las llamadas condicionalidades. La Comisión (siempre de acuerdo con el BCE y, eventualmente, con el FMI) sigue los avances de la realización del programa y de las condicionalidades. La monitorización puede dar lugar a cambios y a actualizaciones del mismo programa (dependiendo también de eventuales inconvenientes de la situación económica) o, en caso de incumplimiento, previa decisión del Consejo, al corte de las financiaciones.

\section{Dos posibles interpretaciones}

Como era de esperarse, el nuevo escenario institucional se ha caracterizado por muchísimas interpretaciones ${ }^{22}$, que han profundizado en aspectos importantes de las transformaciones sufridas. Sin embargo, tal vez dos conceptos elaborados por Michel Foucault -el pastorado y el poder disciplinario- puedan ser útiles para ayudar a comprender, desde otra perspectiva, algunas de las dinámicas actuales. Por lo general, se trata de dos técnicas de gobierno de las personas físicas que, sucesivamente, estarían a cargo de los Estados y, más en específico, de los ejecutivos nacionales (en menor medida de sectores de los parlamentos y de las élites nacionales).

20. K. Tuori, K. Tuori, The Eurozone Crisis, p. 90 ss.

21. Reg. 472/2013 del Parlamento Europeo y del Consejo del 21 de mayo de 2013: M. Ioannidis, "EU Financial Assistance Conditionality after 'Two Pack'” en Zeitschrift für ausländisches öffentliches Recht und Völkerrecht, Heidelberg Journal of International Law, Heidelberg, 2014, pp. 61 ss.

22. G. Martinico, "EU Crisis and Constitutional Mutations: A Review Article", en Revista de Estudios Politicos, Centro de Estudios Políticos y Constitucionales, Madrid, 2014, p. 240 ss. 


\section{El “pastorado económico"}

La nueva gobernanza económica presenta algunos rasgos del pastorado cristiano descrito por Foucault a finales de los años setenta ${ }^{23}$, para indicar un conjunto de tecnologías basadas en un régimen de verdad, dirigidas a asistir, persuadir y orientar los comportamientos de los que dependen de ella en vista de su misma salvación. En las normas europeas antes citadas, una pluralidad de organismos desempeñan funciones pastorales, pero el papel predominante le toca a la Comisión.

La relación entre pastor, oveja, rebaño, verdad y salvación es muy articulada y se traduce en la enseñanza que se desarrolla a través de la observación, la vigilancia, la dirección ejercida en todo momento ${ }^{24}$. En nuestro caso, la verdad adquiere la función del expertise - una serie de principios y nociones atribuibles a una determinada doctrina económica- que constituye la base de los complicadísimos dispositivos de vigilancia sobre las políticas económicas y de balance de los Estados. Además, desde una óptica más sutil, por un lado el pastor dirige, de forma general y permanente, las conciencias de las ovejas; y por otro lado, les extrae algunas verdades ${ }^{25}$, es decir, él debe conocer a la perfección a cada oveja. A este propósito, es suficiente recordar los continuos encuentros y los documentos económicos que los Estados deben someter de forma periódica a la Comisión (programas de estabilidad, programas nacionales de reforma, programas de balance nacionales a mediano plazo). De esta forma, el control es continuo, seguido y de tendencia ilimitada, y se parece mucho a una especie de "examen de conciencia" del que todo gobierno nacional debe hacerse cargo frente a la Comisión.

Esta práctica posibilita una forma de dirección que podría resumirse en la fórmula "quiero que el otro me diga lo que debo querer", que se diferencia de la dirección en sentido jurídico (cfr. infra) ${ }^{26}$. De ahí que el pastor no sea "el hombre de la ley"27, sino más bien el médico que debe hacerse cargo de cada situación, prestando el auxilio necesario. Está claro que cuanto peores son las condiciones de una oveja (o del rebaño), más intensas deben ser las curas pastorales.

Por lo tanto, el poder pastoral tiene una naturaleza individualizante, porque tiene el objetivo de construir un sujeto que, "sometido" a un régimen de verdad (es decir, al expertise), acepta la autoridad del pastor para su salvación: la obediencia sirve para

\footnotetext{
23. M. Foucault, Sécurité, territoire, population, Gallimard-Seuil, Paris, 2004, p. 119 ss. 24. Ibid., p. 184.

25. Más analíticamente, véase M. Foucault, Du governement des vivants, Gallimard- Seuil, Paris, 2012. 26. Ibid.

27. M. Foucault, Sécurité, territoire, population, p. 177.
} 
preparar al individuo para renunciar a su propio egoísmo e interés ${ }^{28}$. Por consiguiente, la acción del pastor siempre será coyuntural e individual, porque cada oveja no puede ser tratada igual que otras ${ }^{29}$. Para hacer eso, él debe servirse de una "detallada economía de los méritos y de los deméritos" ${ }^{\text {. }}$. Con respecto a nuestro argumento, pensemos en cómo la Comisión gestiona la evaluación de los posibles desequilibrios macroeconómicos de los Estados miembros. En el ámbito del "mecanismo de alerta" ${ }^{31}$ y sobre la base de una serie de indicadores, analiza pormenorizadamente todas las situaciones económicas nacionales para comprobar su buen funcionamiento. Las que presenten resultados preocupantes son sometidas a un "examen exhaustivo". Además, dependiendo de los resultados económicos del año anterior, la Comisión ha establecido seis clases (a las que corresponden otros tantos niveles de monitorización y específicas acciones), en las que cada año son insertados los Estados, con la opción de mejorar o empeorar su posición (dependiendo de cómo se hayan portado) ${ }^{32}$.

Además, el poder pastoral es altruista -el pastor siempre cuida de los demás y nunca de él mismo- porque lo ejerce por el exclusivo interés de su destinatario. Todo eso se concreta en el control sobre quien puede hacerse daño ${ }^{33}$. En el contexto europeo, la naturaleza oblativa de esas funciones se destaca también en los pareceres y en las recomendaciones (también estos formulados por la Comisión) dirigidos a cada Estado, por ejemplo, en relación con los documentos que estos deben preparar periódicamente; se trata de pareceres y de recomendaciones cuya finalidad es poner en guardia, solicitar, animar, basándose en consideraciones de carácter macroeconómico, en la mejora de las condiciones del Estado destinatario y en la relativa adecuación de sus políticas.

El pastor actúa al mismo tiempo como omnes et singulatim. Por un lado, él tiene la tarea de salvar al rebaño, incluso con elecciones extremas: la oveja motivo de escándalo, aquella corrupta que puede corromper a todo el rebaño debe ser abandonada, tal vez excluida $^{34}$. Por otro lado, cada oveja necesita del mayor cuidado. Por consiguiente, al

28. De forma parcialmente semejante, cfr. J.H.H. Weiler, La Costituzione dell'Europa, Bologna, 2003, p. 511 ss., sobre todo p. 521 ss.

29. M. Foucault, Sécurité, territoire, population, p. 177.

30. Ibid, p. 176.

31. Arts. 3 ss., reg. 1176/11.

32. Esas clases son: 1) No subsistencia del desequilibrio; 2) desequilibrio que exige una monitorización y una acción política; 3) desequilibrio que exige una monitorización y una fuerte acción política; 4) desequilibrio que exige una monitorización específica y una fuerte acción política; 5) desequilibrio excesivo que exige una monitorización específica y una fuerte acción política; 6) desequilibrio excesivo que exige una fuerte acción política y el inicio del procedimiento por desequilibrio excesivo. Cfr. http://ec.europa.eu/economy_finance/economic_governance/macroeconomic_imbalance_procedure/ mip_reports/index_en.htm.

33. M. Foucault, Sécurité, territoire, population, p. 131.

34. Ibid, p. 172. 
pastor se le reconoce el mérito de luchar sin freno contra los peligros y de rescatar a las ovejas, sobre todo cuando son indómitas ${ }^{35}$. También esta doble naturaleza parece encontrarse en la gobernanza económica europea en la cual la Comisión (y el Consejo en sus diferentes formas) siempre debe prestar atención a la general situación económica de la Unión y a la de cada Estado. A este propósito, reaparece el mecanismo de alerta que sirve para detectar desequilibrios económicos de cada Estado que puedan comprometer el funcionamiento de la unión económica y monetaria (o de la UE) en su globalidad ${ }^{36}$.

Ya no es necesario profundizar en el tema, solo cabe destacar que el centro de todo es el sujeto, el Estado (mejor dicho, sus organismos nacionales de gobierno) que, por un lado, está obligado a obedecer a la enseñanza pastoral y que, por otro lado, respecto de los ciudadanos, es el único responsable de sus políticas económicas (y sociales). De ahí que asistamos a una inversión de responsabilidad: también frente a resultados negativos, el inspirador de las políticas sigue estando exento de cualquier forma de responsabilidad política hacia los electores de los Estados directamente interesados. De hecho, la salvación no depende del pastor, que solo puede actuar con la máxima diligencia sin poder asegurarla totalmente.

\section{El poder disciplinario y la ayuda financiera}

La incidencia en la esfera de los Estados que se benefician de ayuda financiera da lugar a una dinámica que se parece mucho a una especie de poder disciplinario de la soberanía estatal (o, más precisamente, de las clases nacionales de gobierno). Foucault se ha ocupado del poder disciplinario también desde la óptica de esencia institucional y con respecto al cuerpo humano ${ }^{37}$. Sin embargo, algunas de sus intuiciones pueden ser útiles para aclarar algunos de los rasgos que ha ido adquiriendo el ordenamiento europeo. De manera diferente pero más intensa que el pastorado, el poder disciplinario representa la aplicación de una específica ciencia en algunos individuos para fortalecerlos, por medio de una práctica gradual y duradera. Los relativos dispositivos tienen una naturaleza latamente terapéutico-pedagógica, porque están dirigidos a "amansar" los cuerpos y las almas con reglas determinadas ${ }^{38}$. Ellos actúan por medio de los siguientes mecanismos: 1) la reglamentación analítica de los comportamientos; 2) la "vigilancia

35. Ibid, p. 175.

36. Artículos 2 y 3, Reg. 1176/11.

37. M. Foucault, Surveiller et punir. Naissance de la prison, Gallimard, Paris, 1975, sobre todo la tercera parte; Id., Le pouvoir psychiatrique, Gallimard-Seuil, Paris, 2003, sobre todo pp. 41-122.

38. Véase G. Deleuze, Foucault, Cronopio, Napoli, 2009, p. 43. 
jerárquica”, que sirve para permitir la permanente e integral observación, además del análisis de los individuos sometidos; 3) la "sanción normalizadora", que se basa en el binomio sanción/satisfacción en relación con la aceptación o la violación, por parte del sujeto, de las reglas disciplinarias ${ }^{39}$; la desviación del estándar implica intervenciones correctivas para asegurar que la acción del sujeto mismo se adapte a la norma.

De ahí que la norma sea central. Foucault ${ }^{40}$ la concibe como un mecanismo que afirma, a través de una serie de tecnologías, lo normal (normalización) ${ }^{41}$, es decir, constituye un modelo de comportamiento que el sujeto, gracias al ejercicio, interioriza y adquiere como propio de forma que, de un momento a otro, la disciplina empiece a funcionar por sí sola ${ }^{42}$. Por lo tanto, la norma indica la oposición entre lo normal y lo anormal, puesto que tiene el objetivo de homogeneizar (y convertir en eficientes) a los individuos sometidos al régimen disciplinario.

Volviendo a la experiencia europea, en los mecanismos de asistencia financiera, se hallan muchos aspectos de este paradigma. Para saber más es suficiente leer los MoU, es decir, los informes de inspección de la Comisión, del Fondo Monetario Internacional y del Banco Central Europeo, los informes relativos a las condicionalidades por parte de los Estados receptores ${ }^{43}$. Por ejemplo, en el caso de la República Helénica, el primer memorándum se refiere a los numerosos ámbitos de intervención, describiendo pormenorizadamente las medidas para adoptar, las modalidades y los tiempos de ejecución, siempre bajo el control continuo de la llamada Troika, que debe verificar la puesta en práctica correcta en relación con el pago de la siguiente cuota de préstamo.

Más allá de esas semejanzas, hay otro aspecto para destacar: la finalidad productiva de los mecanismos de asistencia. El concepto de ownership del Estado beneficiario aclara ese aspecto, por indicar la voluntaria asunción de responsabilidades, por parte del Gobierno nacional interesado, de un programa de políticas económicas (correctivas) que sea realizable y que corresponda al interés exclusivo del Estado mismo ${ }^{44}$. Se trata de un programa que, por su dificultad (por ejemplo, en términos de oposición social), el Gobierno no emprendería de forma espontánea ${ }^{45}$. Por lo tanto, los sistemas de asistencia

\footnotetext{
39. M. Foucault, Surveiller et punir, p. 180 ss.

40. Véase también G. Canguilhem, Le normal et le pathologique, Puf, Paris, 2013.

41. P. Macherey, La force des normes: de Canguilhem a Foucault, La Fabrique, Paris, 2009.

42. M. Foucault Le pouvoir psychiatrique, p. 41 ss.

43. Esos documentos se pueden encontrar en el enlace: http://ec.europa.eu/economy_finance/assistance_eu_ms/intergovernmental_support/index_en.htm.

44. Cfr. FMI, Strenghtening Country Ownership of Fund-Supported Programs, 2001, 6.

45. FMI, Strenghtening Country Ownership, cit., p. 7 ss., además de J. M. Boughton, Who's in Charge? Ownership and Conditionality in IMF-Supported Programms, International Monetary Fund, WP/03/191, 2003; y M. Ioannidis, EU Financial Assistance Conditionality after "Two Pack", p. 89 ss.
} 
exigen una práctica, es decir, que el Estado con problemas acepte seguir (por su mismo provecho), con la ayuda, bajo la vigilancia y con la autorización de expertos, un camino de reformas económicas, definido con extremado análisis (tanto en los contenidos como en los tiempos) y ajustado a los estándares disciplinarios (y a las relativas premisas económicas). La naturaleza voluntaria y, al mismo tiempo, disciplinaria, de estos mecanismos financieros dificulta su análisis en términos jurídicos (cfr. infra).

Tampoco debemos olvidar que este sistema de poder sirve para producir clases de gobierno que, apenas terminen los vínculos derivados del programa de ayuda, siguen persiguiendo "una política de balance virtuosa"46. Esto lo destacamos con claridad del reg. 472/13, que contiene normas relativas a la fase posprograma (art. 14) cuya tarea (además de tutelar las necesidades de los acreedores) también es verificar que la disciplina funcione realmente sin ninguna ayuda. También en este caso, el sujeto desempeña el papel central, lo que lleva a aquella inversión de responsabilidad de la que hablamos antes.

\section{El papel del derecho en la nueva gobernanza económica}

Estos dos conceptos ayudan a comprender algunas dinámicas de la UE. Por lo que al pastorado se refiere, cabe observar que las normativas europeas citadas han llevado a la radicalización del método abierto de coordinación que caracterizó a la primera fase de la Unión económica y monetaria. Hoy día, ellas permiten que las Instituciones ejerzan su influencia en las políticas nacionales (sobre todo de los países con mayores problemas) mucho más que en el pasado ${ }^{47}$. Todo eso lleva al funcionamiento de las políticas económicas y de balance nacionales, con la consiguiente juridicidad de conceptos muy vagos relacionables con la idea de "bienestar económico" de los Estados miembros. Se trata de un objetivo que, en el pasado, se remitía al proceso político-democrático nacional en el ámbito de la coordinación del Consejo. Sin embargo, esa juridicidad es contradictoria. Por un lado, ella se presenta como una textura muy tupida que envuelve los comportamientos macroeconómicos y fiscales de los Estados. Por otro lado, la normativa (sobre todo) derivada da lugar a "una deslegalización reptante", que abre las puertas a la evaluación política discrecional de los sistemas tecnocráticos posdemocráticos ${ }^{48}$.

46. A este concepto remite, por ejemplo, el Tribunal de Justicia en la Sentencia C-370/12, $\$ 135$.

47. A. Somek, "Delegation and Authority: Authoritarian Liberalism Today", en European Law Journal, 2015, pp. 340-360, sobre todo, pp. 342-343.

48. C. Joerges, S. Giubboni, Diritto, politica ed economia nella crisi europea, en G. Allegri, G. Bronzini (eds.), Il tempo delle costituzioni, Manifestolibri, Roma, 2014, p. 119. 
La técnica del pastorado convierte en compatibles a esos dos aspectos, gracias a la adaptación de la regla de comportamiento que cada Estado debe seguir en el caso específico. Sin embargo, de esa manera el alcance del código jurídico solo se limita a los aspectos procedimentales de esos procesos decisionales, puesto que todo lo demás se remite a complicadísimas evaluaciones técnico-económicas. Por lo tanto, mientras la reglamentación jurídica de origen publicista se desarrolla sobre la base del binomio conformidad/divergencia (de comportamientos y prácticas) respecto de una norma jurídica (es decir, sobre la base del juicio legítimo/ilegítimo), en cambio, la nueva gobernanza atañe, por lo general, a evaluaciones, es decir, al binomio éxito/fracaso de las prestaciones económicas de los Estados y de sus políticas (programadas o implementadas $)^{49}$.

Si las evaluaciones de la Comisión atañen a los resultados económicos de un Estado, esto implica la institución del mercado como "lugar de verificación y de evaluación de credibilidad" de los gobiernos nacionales ${ }^{50}$, porque el "Estado deudor democrático" debe gozar de la confianza de los mercados (y, claro está, de sus acreedores) ${ }^{51}$. Y si pensamos en que la evaluación de las políticas públicas es definida como un proceso de aprendizaje, entonces queda confirmado que las normas sobre la gobernanza económica han llevado a un sistema basado más en un conjunto de conocimientos gestionado por el pastor que en la eficacia jurídica. Examinando los préstamos realizados o programados, el pastor ayuda, fomenta, advierte ( $y$, cuando sea necesario, amenaza) al Estado para que persiga políticas exitosas. Así las cosas, se trata de dispositivos dirigidos a movilizar los recursos internos del sujeto para alcanzar un determinado estándar de acción y específicos resultados económicos.

Lo anterior conduce al tema de las relaciones entre hard law y soft law en este ámbito. Los instrumentos con escasa legalización (por ejemplo, pareceres y recomendaciones del Consejo y de la Comisión) son los más útiles para realizar un proyecto institucional basado en la autoreglamentación y autorresponsabilidad de cada Estado, en el cual el pastor no debe rendir cuentas de las relativas elecciones a los ciudadanos directamente interesados. En efecto, quien formula el mando jurídico se hace cargo de la relativa responsabilidad, remitiendo al destinatario solo una obligación de ejecución; es lo que Foucault ha dicho respecto del poder jurídico, que decide en lugar de todos, imponiendo

49. L. Bazzicalupo, Il governo delle vite. Biopolitica ed economia, Laterza, Roma-Bari, 2006.

50. Ibid., p. 46.

51. W. Streeck, Buying Time The Delayed Crisis of Democratic Capitalism, Verso Books, London - New York, 2014. Véase también Tribunal de Justicia, C-370/12, \$135. 
su voluntad a pesar de la de los demás ${ }^{52}$. De ahí que nos expliquemos por qué esta reglamentación no se fundamenta en el "sistema de la ley", sino en el de la salvación, es decir, en la virtud del sujeto ${ }^{53}$. Por lo demás, la flexibilidad de esos instrumentos está relacionada con la naturaleza contingente $y$, al mismo tiempo, permanente de la evaluación. Ella deriva del hecho de que su resultado puede cambiar continuamente, dependiendo de las performance de cada Estado y de eventuales factores externos ${ }^{54}$.

Incluso, por lo que a los programas de acuerdo se refiere, la lógica jurídica parece ser residual. Esto lo podemos demostrar recordando la incertidumbre relativa a los efectos jurídicos de los MoU. De hecho, hay dudas acerca de si ellos son jurídicamente vinculantes o si, en cambio, son programas políticos suscritos por los Gobiernos beneficiarios $^{55}$. Sin embargo, cabe destacar que los programas de arreglos prevén la partición del pago del préstamo y -como hemos dicho antes- el desbloqueo de cada parte depende de una evaluación positiva de la Comisión sobre la correcta y oportuna puesta en práctica de los Memorándums. Así las cosas, debería estar claro que la ejecución de las condicionalidades no deriva (solo) de su carácter obligatorio, sino más bien de la situación de necesidad económica del Estado en cuestión. También en este caso la lógica disciplinaria prevalece sobre aquella jurídica, basada como está en el binomio sanción/satisfacción en relación con la aceptación o la violación de las normas disciplinarias.

A ello se debe que la gobernanza económica se traduzca en una compleja, difundida y difusiva presión ejercida sobre $\operatorname{los} \operatorname{Estados}^{56}$ para inducirlos a seguir determinadas políticas económicas. Esas intervenciones normativas han institucionalizado un régimen de saber-verdad (y de poder) que, de hecho, es incontestable. Por lo tanto, la emergencia debida a la crisis ha llevado a la formación de un muy difundido sentido común a ese respecto. Se ha realizado, pues, una gran ejemplificación valorativa de por qué determinados objetivos económicos deben prevalecer sobre los demás valores. En definitiva, la nueva gobernanza económica persigue el objetivo de "normalizar" las políticas de los Estados miembros, es decir, de superar situaciones económicas patológicas a través de la adhesión a determinadas reglas (la "frugalidad" del Estado), las únicas que pueden asegurar la curación.

52. M. Foucault, Du governement des vivants, cit.

53. Ibid.

54. L. Bazzicalupo, Il governo delle vite, cit.

55. Véase, entre otros, A. Baraggia, "Conditionality Measures within the Euro Area Crisis: A Challenge to the Democratic Principle?, en Cambridge Journal of International and Comparative Law, 2015, pp. 268-288, sobre todo, pp. $276-277$.

56. L. Bazzicalupo, "Le mobili linee di confine nella normatività sociale e la indeterminatezza delle procedure", en A. Tucci (ed.), Disaggregazioni. Forme e spazi di governance, Mimesis, Milano, 2013, pp. 29 ss. 
Cabe aclarar este aspecto. A pesar de que esas reglas tengan una naturaleza técnica, ellas remiten a una precisa doctrina económica que ha sido insertada de manera progresiva en la Constitución Económica Europea. Por consiguiente, si bien es verdad que las negociaciones políticas están permitidas, ellas deben respetar los límites impuestos por esa doctrina. Por un lado, esto explica por qué también las decisiones tomadas a nivel intergubernamental se expresan, sobre todo, con un lenguaje económico (por ejemplo, la admisión a los programas de arreglos); por otro lado, esto aclara por qué -también a ese nivel- se emplean rígidas normas de procedimiento (por ejemplo, respecto de la mayoría inversa) que limitan el alcance de la negociación. Por esa razón, el discurso político a veces es extremo. Tal y como lo demuestran los sucesos griegos, el argumento político -si no está conforme a la verdad-puede desembocar con facilidad en el tema de la salida de la eurozona, es decir, en el problema de la revocación de la confianza en el pastor o de la revocación de la confianza en el Estado miembro ${ }^{57}$. Esta es una consecuencia de la escasa relevancia de la voice, de por sí incompatible con las lógicas pastorales y disciplinarias ${ }^{58}$.

Todo esto pone de manifiesto la gran diferencia de este modelo con respecto al de la “integración del derecho". En efecto, en este caso el pluralismo institucional y los relativos conflictos no se solucionan con procedimientos legales. Pensemos en la exclusión del procedimiento de infracción (y, pues, de las posibilidades de compromiso con este relacionadas) por los déficits excesivos ${ }^{59}$ a favor de las actividades de evaluación y de los eventuales poderes sancionatorios (que el derecho derivado hace aumentar) de las instituciones; pensemos también en los límites de las competencias del Tribunal de Justicia previstas por el Fiscal Compact en relación con los incumplimientos de los Estados ${ }^{60}$. Además, desatendiendo el principio de igualdad de los Estados, la actual gobernanza económica ha producido una jerarquía entre estos, y nos lo confirma también el hecho de que los sistemas nacionales siempre mencionan la competitividad, además de las confrontaciones entre deudas públicas y de los indicadores que la Comisión debe utilizar para velar por los balances públicos o los desequilibrios macroeconómicos.

57. Cfr., por ejemplo, la declaración de la Cumbre del Euro del 12 de julio de 2015 (SN 4070/15).

58. Cfr. también J. H .H. Weiler, La Costituzione dell'Europa, cit., p. 33 ss.

59. Artículo 126, párr. 10 TFUE. Cfr., por ejemplo, K.A. Armstrong, The New Governance of EU Fiscal Discipline, p. 612 ss.

60. Artículo 3, apartado 2 del Tratado en mención (artículo 8). Véase, entre otros, R. Dehousse, The "Fiscal Compact": legal uncertainty and political ambiguity, disponible en http://www.institutdelors.eu/media/fiscalpact_r.dehousse_ne_feb2012. pdf?pdf=ok. 


\section{Consecuencias para la actividad de los jueces}

Está claro que esta situación no solo debilita el principio democrático, sino también el del rule of law que, como hemos dicho, es la base del ordenamiento jurídico europeo. Por lo tanto, esta situación afecta también a los jueces europeos y nacionales. En relación con los primeros, cabe recordar la autoeliminación puesta en práctica por el Tribunal de Justicia con referencia a los Estados que se benefician de asistencia económica. Por ejemplo, el Tribunal del Trabajo de Oporto pidió al Tribunal de Justicia que interpretara el artículo 31 de la Carta de los Derechos Fundamentales para verificar la legitimidad de algunas medidas anticrisis relativas al trabajo público promulgadas por Portugal en el programa de asistencia. El Tribunal negó su competencia porque en la ordenanza de remisión no había "ningún elemento concreto que permitiera creer que esa ley sirviera para poner en práctica el derecho de la Unión” ${ }^{61}$. Ocurrió lo mismo con ocasión de procedimientos jurisdiccionales análogos ${ }^{62}$. Si bien la decisión asombra por el exceso de formalismo, es coherente con la naturaleza disciplinaria de los programas de asistencia. De hecho, basándose en la movilización de las energías propias del sujeto, ellos parecen difícilmente relacionables con el derecho de la Unión. Sin embargo, cabrá verificar si el reg. 472/13 llevará a reconsiderar esas afirmaciones.

Por lo que a los jueces estatales se refiere, se pueden distinguir dos tipos de senten$\operatorname{cias}^{63}$. Las primeras - para definir una controversia- toman en consideración también los efectos que ellas mismas pueden producir sobre la situación económica del país. Por ejemplo, el Consejo de Estado griego ha considerado legítimas una serie de medidas de implementación del primer Memorándum en materia social, por la gravedad de la crisis y de la consiguiente prevalencia del interés público nacional y europeo ${ }^{64}$. En este caso, el juez no solo empleó criterios de sostenibilidad económica, sino sobre todo esos parámetros prevalecieron sobre la tutela de los derechos. Este realismo, probablemente ahora apoyado por las normas nacionales (preferiblemente de valor constitucional) de recepción del Fiscal Compact, está bastante difundido. Sin embargo, el pastor no se ocupa de esas sentencias porque no ponen en discusión (es más, sirven para) la salvación.

\footnotetext{
61. Tribunal de Justicia, C-264/12 (\$19).

62. Cfr. Tribunal de Justicia, C-565 y 566/13, y antes C-128/12.

63. C. Kilpatrick, B. De Witte (ed.), "Social Rights in Times of Crisis in the Eurozone: The Role of Fundamental Rights' Challenges”, en Law Working Papers, European University Institute, Badia Fiesolana, 2014/05.

64. Consejo de Estado griego, Decisión 668/2012; E. Psychogiopoulou, "Welfare Rights in Crisis in Greece: The Role of Fundamental Rights Challenges" y M. Yannakourou, "Challenging austerity measures affecting work rights at domestic and international level. The case of Greece”, ambos en Social Rights in Times of Crisis, cit., p. 5 ss. y p. 19 ss. respectivamente.
} 
Sin embargo, algunos jueces se han limitado a tutelar los derechos de los ciudadanos, prescindiendo de las consecuencias económicas de sus decisiones. En estos casos, si se trata de sentencias importantes desde el punto de vista financiero, el pastor se siente implicado y emplea esas decisiones para formular una evaluación económica desfavorable del país o, por lo menos, para aumentar las curas pastorales. Dos ejemplos pueden ayudarnos a comprenderlo mejor.

El primero atañe otra vez a Portugal ${ }^{65}$. A propósito de la sentencia del Tribunal Constitucional portugués n. ${ }^{\circ} 187$ de abril de 2013 que anuló una serie de medidas de balance dirigidas a reducir el gasto público ${ }^{66}$, la Comisión europea dijo:

A necessary condition or the country's return to market financing will be the government's reaffirmed ownership and resolute implementation of the programme. However, the risks from further negative rulings by the Constitutional Court cannot be discarded and could make the government's plans to fully access the debt market from mid-2014 on significantly more challenging ${ }^{67}$.

Además, la decision del Tribunal Constitucional "raised further doubts about the government's capacity to push through the necessary reforms. As a consequence, investors demanded higher premiums to reflect increased sovereign risk and Portuguese bond yields decoupled from other European sovereigns" ${ }^{68}$. Con respecto a algunas medidas en materia de revisión del gasto público que, en aquel entonces, se estaba aprobando, la Comisión advertía que si algunas de ellas hubieran sido declaradas inconstitucionales, el Gobierno, para alcanzar los objetivos concertados, habría tenido que volver a formular el balance público, pero esto "in view of the rapidly shrinking room of manoeuvre in identifying appropriate consolidation measures this would imply increasing risks to growth and employment and would reduce the prospects for a sustained return to financial markets" ${ }^{69}$.

65. M. Nogueira de Brito, Putting Social Rights in Brackets? The Portuguese Experience with Welfare Challenges in Times of Crisis, J. Gomes, Social Rights in Crisis in the Eurozone. Work Rights in Portugal y R. Cisotta, D. Gallo, The Portuguese Constitutional Court Case Law on Austerity Measures: A Reappraisal, todos en Social Rights in Times of Crisis, p. 57 ss., p. 78 ss. y p. 85 ss. respectivamente.

66. Sentencia del Tribunal Constitucional portugués 187 del 2013; G. Coelho, P. C. de Sousa, La Morte Dei Mille Tagli, en Giornale di diritto del lavoro e delle relazioni industriali, 2013, p. 527 ss.

67. Comisión Europea, The Economic Adjustment Programme for Portugal. Eighth and Ninth Review, Occasional Papers 164, noviembre de 2013, pp. 6 y 44, disponible en http://ec.europa.eu/economy_finance/publications/occasional_paper/2013/ pdf/ocp164_en.pdf.

68. Comisión Europea, The Economic Adjustment Programme for Portugal. Eighth and Ninth Review, p. 43.

69. Ibid., p. 20. 
Por lo tanto, la Comisión hace recaer sobre el Tribunal Constitucional el riesgo de desestabilizar la resistencia económica del sistema y, sobre todo, de desacreditar al Gobierno frente a los inversores, con posibles consecuencias sobre las tasas de interés de la deuda pública. Y esto ocurre al desatender los principios fundamentales del Estado liberal democrático, como aquel de la separación de los poderes y de la independencia de los jueces.

El segundo ejemplo atañe a la sentencia del Consejo de Estado griego que, en 2015, declaró inconstitucional la reforma de las pensiones aprobada en $2012^{70}$. A ese propósito, en la declaración de la Cumbre del Euro del 12 de julio de 2015, se afirmó que, para acabar con el $M o U$ (relativo al tercer programa de ayudas), el Gobierno griego debía comprometerse también:

To carry out ambitious pension reforms and specify policies to fully compensate for the fiscal impact of the Constitutional Court ruling on the 2012 pension reform and to implement the zero deficit clause or mutually agreeable alternative measures by October $2015^{71}$.

Estos ejemplos demuestran que, por esa lógica de gobierno, solo el mercado y sus “instituciones fiduciarias" ( $y$, más en general, solo una determinada doctrina económica) pueden emitir un juicio sobre las acciones económicas de los Gobiernos, mientras que no puede hacerlo el juez constitucional administrativo. En definitiva, estamos asistiendo al ocaso del principio del rule of law, debido a fuertes e inéditas presiones que tienden a imponer la lógica económica también al poder judicial.

\section{Conclusiones}

Puesto que la situación producida por la nueva gobernanza económica europea conlleva una serie de problemas desde diferentes puntos de vista, en junio de 2015 se produjo un ambicioso informe sobre su reforma ${ }^{72}$. El documento profundiza en una

\footnotetext{
70. Cfr. los informes en Keep Talking Greece, "Supreme Court orders Greece to reverse 2012 pension cuts as 'unconstitutional”, disponible en http://www.keeptalkinggreece.com/2015/06/11/supreme-court-orders-greece-to-reverse-2012-pension-cuts-as-unconstitutional y en R. Godin, "Grèce les coupes dans les retraites jugees inconstitutionnelles", La Tribune, disponible en http://www.latribune.fr/economie/union-europeenne/grece-les-coupes-dans-les-retraites-jugees-inconstitutionnelles-483130.html.

71. Declaración de la Cumbre del Euro del 12 de julio de 2015, 3.

72. J. C. Juncker, D. Tusk, J. Dijsselbloem, M. Draghi y M. Schulz (eds.), Completing Europe's Economic and Monetary Union, junio de 2015.
} 
serie de cuestiones de las que cabría ocuparse en el corto, mediano y largo plazo. Aquí cabe destacar dos iniciativas para implementar en el corto plazo. Para que haya mayor acuerdo entre los Estados miembros, el documento propone la formación, a nivel nacional, de autoridades independientes en competitividad, con la tarea de afirmar, por ejemplo, "whether wages are evolving in line with productivity and compare with developments in other euro area countries and in the main comparable trading partners" o los progresos "made with economic reforms to enhance competitiveness more generally"73. Esas autoridades deberían constituir una red a nivel europeo; además, se deberían evaluar mejor los desequilibrios macroeconómicos, para implementar las reformas y que sirvan también para el área euro en su globalidad ${ }^{74}$.

En segundo lugar, el informe también se ocupa del tema de la responsabilidad de las políticas de balance nacional. A este propósito se propone la institución de un European Fiscal Board, como órgano consultivo de coordinación y ayuda de los consejos fiscales nacionales, con la tarea de evaluar los balances nacionales (y la relativa ejecución) en relación con los objetivos propuestos ${ }^{75}$. Este organismo "should form an economic, rather than a legal, judgement on the appropriate fiscal stance, both at national and euro area level, against the background of EU fiscal rules"76. La Comisión ha instituido de repente ese organismo ${ }^{77}$.

No podemos analizar en detalle ese informe. Las dos propuestas que acabamos de mencionar ponen de manifiesto que el informe aspira a incrementar -a pesar de sus límites- el modelo pastoral (por ejemplo, el aumento de las evaluaciones de los desequilibrios macroeconómicos). Incluso podemos hablar de una colonización pastoral de los ordenamientos estatales. De hecho, las autoridades nacionales de competitividad representan la transposición a nivel interno de la lógica de evaluación de las políticas públicas. Lo mismo ocurre con el European Fiscal Board, que debería reforzar el papel de organismos independientes de balance ya presentes en el derecho vigente.

No es este el momento de pensar en soluciones alternativas. Sin embargo, podemos desear que cualquier reforma de la gobernanza económica no se dé por modelos abstractos, sino que considere las diferencias económicas, históricas y culturales de los

\footnotetext{
73. Ibid., p. 8 .

74. Cfr. Comisión Europea, Recomendación del Consejo sobre la institución de comités nacionales para la competitividad en la eurozona, COM (2015) 601 final.

75. Completing Europe's Economic and Monetary Union, p. 14.

76. Ibid, p. 23.

77. Decisión de la Comisión 2015/1937 del 21 de octubre de 2015, que instituye un comité consultivo independiente europeo para las finanzas públicas.
} 
Estados (de la eurozona) $)^{78}$. Además, es necesario que, a nivel europeo, se realice una clara y sólida juridización de los derechos sociales (entendidos en sentido amplio). En efecto, sobre todo en los países más afectados por la crisis, esos derechos han sucumbido frente a la lógica de la salvación económica. De ahí que sea necesario definir (de forma jurídicamente vinculante) una serie de derechos sociales de los que todos los ciudadanos de la Unión puedan gozar al mismo tiempo ${ }^{79}$. Más en general, parece indispensable reflexionar sobre soluciones institucionales que le permitan al proceso democrático (a nivel nacional y europeo) disputarse con la racionalidad económica su dominio exclusivo sobre el gobierno de los Estados y de la Unión. Esto significaría volver a pensar en la fuente de los mecanismos de gestión de la moneda única, que deberían volver a relacionarse con las realidades sociales de los Estados miembros ${ }^{80}$. No obstante, el problema es verificar si estos deseos son compartidos por la mayoría de los electores europeos.

Traducción del italiano de M. Colucciello

78. W. Streeck, Buying Time.

79. Por ejemplo, F. W. Schar, “After the Crash: A Perspective on Multilevel European Democracy”, en European Law Journal, 2015, (21) 3, pp. 384 ss., sobre todo p. 400.

80. W. Streeck, Buying Time. 